\title{
FEA OF FRICTIONAL CONTACT PROBLEMS USING NAGATA PATCHES FOR SURFACES DESCRIPTION
}

\author{
D. M. Neto ${ }^{1}$, M. C. Oliveira ${ }^{1}$, L. F. Menezes ${ }^{1}$, J. L. Alves ${ }^{2}$
}

${ }^{1}$ CEMUC, Mechanical Engineering Center of the University of Coimbra, Department of Mechanical Engineering, University of Coimbra, Portugal (diogo.neto@dem.uc.pt)

${ }^{2} \mathrm{CT} 2 \mathrm{M}$, Center for Mechanical and Materials Technologies, Department of Mechanical Engineering, University of Minho, Portugal

\begin{abstract}
The numerical simulation of problems involving contact with friction between deformable and rigid bodies is highly dependent of the predicted contact conditions, which are continuously changing during the process. In the case of sheet metal forming processes, the forming tools are assumed to behave rigidly, and thus only the definition of the outer surfaces is required for the simulation. This paper presents the contact search algorithms currently implemented in DD3IMP in-house finite element solver, which has been continuously developed and optimized to simulate sheet metal forming processes. Nowadays, in DD3IMP it is possible to describe the forming tools either using Bézier or Nagata patches, for which distinct contact search algorithms are adopted. The results show that the accuracy and computation cost of both algorithms is similar. The Nagata patch description is more versatile than Bézier since it can be extracted using any combination of CAD and mesh generation software. Besides, the numerical results accuracy is almost independent of the polyhedral mesh generated.
\end{abstract}

Keywords: Nagata patch, Tool surface description, Contact search algorithm, DD3IMP.

\section{INTRODUCTION}

Nowadays, the Finite Element Method (FEM) is commonly used to simulate problems involving contact with friction between rigid and deformable bodies. One of the most important topics for attaining accurate FEM results is the treatment of the contact with friction conditions during the process [11]. Most of FEM codes resort to polyhedral meshes for the rigid body surface description due to their wide application, ability to describe complex geometries and simplicity. However, this last characteristic can lead to large errors in the geometrical description and convergence problems in the contact treatment schemes, as well as to an artificial roughness of the surfaces. It is known that smoothing the discretized surfaces can help to obtain optimal and reliable convergence in the numerical simulation procedure, particularly in full implicit FEM codes [4]. However, the smoothing methods typically proposed 
contribute to higher computational cost than the polyhedral mesh description. In this context, a new type of parametric surface was recently proposed by Nagata, which allows smoothing any polyhedral mesh with biquadratic Nagata patches, using only the position and normal vectors at its vertices. Previous studies show that Nagata patch enables the creation of rigid body models much more accurate, both in terms of shape and normal vectors, than those of conventional polyhedral representations [2,6], allowing a more accurate and numerically stable simulation of a wide range of contact with friction processes.

DD3IMP is an in-house finite element solver that has been continuously developed and optimized to simulate sheet metal forming processes [10]. Its main characteristic is the use of a fully implicit algorithm of Newton-Raphson type to solve, within a single iterative loop, the non-linearities related with the frictional contact problem and the elastoplastic behavior of the deformable body [5]. The forming tools are assumed to behave rigidly, and thus only the definition of the outer surfaces is needed for the simulation. Nowadays, in DD3IMP it is possible to describe the forming tools either using Bézier patches or using Nagata patches, defined based on polyhedral mesh models of the outer surfaces. In either case, the master/slave contact strategy combined with the classic node-to-segment algorithm is employed in the code, to enforce the non-penetration condition between the sheet and the tools.

This paper describes the global and local contact search algorithms adopted for both Bézier and Nagata patches. The numerical simulation of a cross tool deep drawing process was selected to validate the new developed algorithms. Hence, section 2 comprises the geometric description of Bézier and Nagata patches, as well the mathematical equivalency between Nagata and biquadratic Bézier patches. The contact search algorithms implemented in DD3IMP are described in section 3, being the local search similar for both types of patches. The accuracy and computational performance of the algorithms is evaluated in section 4 through the numerical simulation of a cross tool shape deep drawing benchmark.

\section{TOOL SURFACE DESCRIPTIONS}

The numerical simulation of problems involving contact with friction between a deformable body and several rigid bodies requires significant care concerning the description of the arbitrarily shaped surfaces that define the rigid bodies. Several schemes have been suggested for tool surface (rigid bodies) description in sheet metal forming analysis, which can be classified in three groups: (i) the analytical function scheme; (ii) the finite element mesh scheme, and (iii) the parametric patch scheme.

The analytical function scheme is very useful to describe simple tool surfaces. Nevertheless its extension for describing complex tool surfaces is limited [18]. In the finite element mesh scheme, the tool surfaces are divided into finite elements which typically present an irregular distribution, leading to computational difficulties in contact searching [14]. Moreover, the elements are usually bilinear which leads to an inaccurate surface description, as well as to an artificial roughness. Nevertheless, currently this method is the most used due to its wide application, ability to describe complex geometries and simplicity. The parametric patch scheme describes the tool surfaces using an assembly of patches. The parametric surfaces typically employed were originally developed for CAD applications, such as NURBS, Bézier 
and Gregory patch $[15,17,12]$. This approach allows using directly the information generate by any CAD system to define the surface geometry. Nevertheless, sometimes this information contains inaccuracies such as geometric discontinuities between patches and inconsistent surface orientations. Moreover, the use of high order polynomials in surface definition involves complex contact search algorithms, leading to high computation cost of the local contact search algorithm [2].

The scheme adopted to define the tool surfaces dictates the algorithm employed to identify, for each node of the deformable body candidate to establish contact, the reference position on the rigid body where contact can be established, called contact search algorithm [14]. In implicit finite element formulations of contact problems, a special attention is required in order to avoid severe convergence problems due to sudden changes of the normal vector to the tool surface [4]. These problems can be overcome using a smoothing contact surface, reproduced through the parametric patch scheme. Additionally, smoothing method provides more accurate approximations of the real shape than the ones obtained with the polyhedral mesh method, particularly in the case of coarse meshes. Therefore, DD3IMP solver adopts a parametric patch description of the tools.

Typically, the sheet metal forming processes involve several forming tools, presenting dissimilar geometries. Therefore, in order to perform the numerical simulation of these processes, each tool must be defined by a set of patches. The necessary number of patches for the proper description of each tool increases with its complexity. Currently, in DD3IMP it is possible to describe the forming tools either using parametric Bézier or Nagata patches. Hence, these two types of parametric patches are described in detail in the next subsections.

\subsection{Bézier patches}

The geometric definition of the surfaces composing the forming tools is usually performed with the aid of CAD software packages. The information about the Bézier patches can be extracted directly from some CAD packages using the standard STEP file format. However, this strategy involves some limitations due to the inherent simplicity of Bézier patches, particularly when applied to describe very complex geometries. Therefore, it is necessary to perform some operations on the CAD model, such as surfaces division, in order to attain a proper geometrical definition with patches presenting a reduced degree.

The most widely used method in geometric modeling applications is the tensor product scheme, which is basically a bidirectional curve scheme $[1,13]$. Thus, a Bézier patch can be defined as the tensorial product of two Bézier curves, given by:

$$
\mathbf{S}(\eta, \zeta)=\sum_{i=0}^{n} \sum_{j=0}^{m} B_{i, n}(\eta) B_{j, m}(\zeta) \mathbf{P}_{i j}, \quad \text { with } \quad 0 \leq \eta, \zeta \leq 1,
$$

where $\mathbf{P}_{i j}$ are the position vector of the vertices of a polygonal control net, leading to a total of $\{(n+1)(m+1)\}$ control points, since the indices $n$ and $m$ present a variation equal to the number of polygon vertices less one, in the $\eta$ and $\zeta$ directions, respectively. The $B_{i, n}(\eta)$ and $B_{j, m}(\zeta)$ are the Bernstein basis functions in the $\eta$ and $\zeta$ parametric directions. The 
Bernstein polynomial of degree $n$ is defined by:

$$
B_{i, n}(t)=\left(\begin{array}{c}
n \\
i
\end{array}\right) t^{i}(1-t)^{n-i}, \quad \text { for } \quad i=0,1, \ldots, n,
$$

where the binomial coefficient is given explicitly by:

$$
\left(\begin{array}{l}
n \\
i
\end{array}\right)=\frac{n !}{i !(n-i) !}, \quad \text { with } \quad 0 \leq i \leq n
$$

where $n$ ! denotes the factorial of $n$. An important feature of Bézier patches is that its geometry does not change neither with the mathematical operations of translation nor with rotation. The translational/rotational of the surface corresponds to subject each control point to the same operation. Figure 1 presents both the polygonal control net and the Bézier patch, in order to highlight the position of the control points that define the patch.

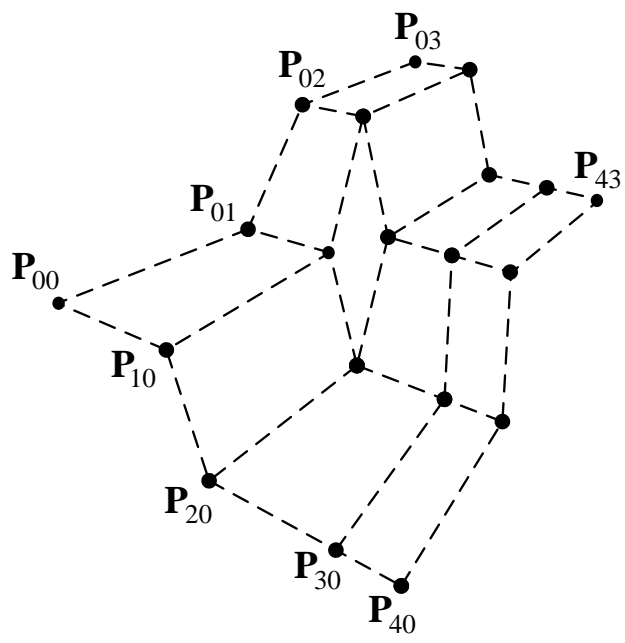

(a)

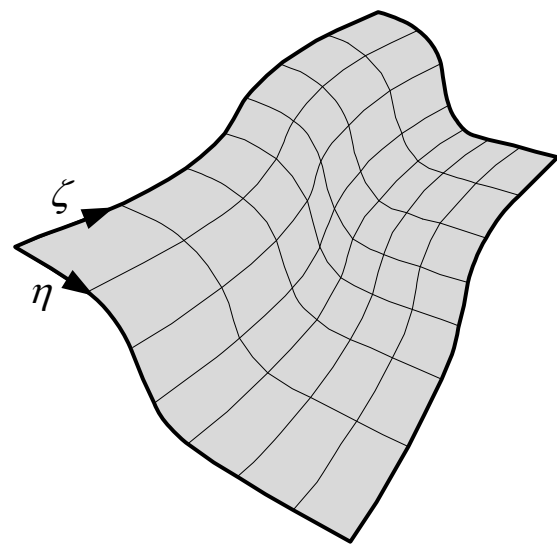

(b)

Figure 1. Example of a quarticxcubic Bézier patch: (a) the control net; (b) the Bézier patch.

Bézier patches can also be described using the monomial form, also called power basis form, which is given by:

$$
\mathbf{S}(\eta, \zeta)=\sum_{i=0}^{n} \sum_{j=0}^{m} \mathbf{b}_{i j} \eta^{i} \zeta^{j}, \quad \text { with } \quad 0 \leq \eta, \zeta \leq 1,
$$

where $\eta^{i}$ and $\zeta^{j}$ are called the basis (or blending) functions and the coefficients of the power basis representation are evaluated using the control points coordinates, as follows:

$$
\mathbf{b}_{i j}=M_{i p} \mathbf{P}_{p r} N_{r j},
$$

where the square matrices $M_{i p}$ and $N_{r j}$, defined with the aid of binomial coefficients previously defined in Equation (4), are described by: 


$$
M_{i p}=(-1)^{p-i}\left(\begin{array}{c}
m \\
p
\end{array}\right)\left(\begin{array}{c}
p \\
i
\end{array}\right) \text { and } N_{r j}=(-1)^{j-r}\left(\begin{array}{l}
n \\
j
\end{array}\right)\left(\begin{array}{l}
j \\
r
\end{array}\right) \text {. }
$$

Note that the definition imposes that $M_{i p}=0$ for $0 \leq i<p$ and $N_{r j}=0$ for $0 \leq r<j$, which results in two upper triangular matrices. The main advantage of the monomial form is the fact that guarantees lower time for operations such as calculations of point coordinates or derivatives. However, this form is numerically less stable, mainly for patches with high degree [1]. Thus, the monomial form presented in Equation (4) is the one used in DD3IMP solver since it is computational less expensive. Nevertheless, in order to avoid numerical instabilities, the maximum patch degree allowed is limited to six, in each parametric direction [9].

\subsection{Nagata patches}

The Nagata patch is a quadratic parametric interpolator for polyhedral meshes. This method can be used as a mesh smoothing technique in order to reduce the sudden changes of the surface normal vector, which characterizes polyhedral meshes composed by bilinear elements [2]. The Nagata patch interpolation algorithm recovers the curvature of surfaces with good accuracy, using only the position and normal vectors of each vertex of the polyhedral model [6]. The strategy followed in DD3IMP solver to describe the forming tools with aid of Nagata patches comprises firstly in a discretization of the tool surfaces using any mesh generator. Note that the discretization of the tools can be achieved using either triangular or quadrilateral bilinear finite elements. Moreover, the required surface normal vectors in each node, of the tool discretization, are evaluated from the information available in the IGES file format, which was originally used in the mesh generation [8].

The Nagata patch is based on the interpolation of an edge using the following curve:

$$
\mathbf{C}(\eta)=\mathbf{x}_{0}+\left(\mathbf{x}_{1}-\mathbf{x}_{0}-\mathbf{c}\right) \eta+\mathbf{c} \eta^{2}, \text { with } \quad 0 \leq \eta \leq 1,
$$

where $\eta$ is the local coordinate of the curve. The position vector of each edge end point is given by $\mathbf{x}_{0}$ and $\mathbf{x}_{1}$, while its unit normal vectors are given by $\mathbf{n}_{0}$ and $\mathbf{n}_{1}$, respectively. The coefficient $\mathbf{c}$, which adds the curvature to the edge is evaluated as:

$$
\mathbf{c}\left(\mathbf{x}_{0}, \mathbf{x}_{1}, \mathbf{n}_{0}, \mathbf{n}_{1}\right)=\left\{\begin{array}{ll}
\frac{\left[\mathbf{n}_{0}, \mathbf{n}_{1}\right]}{1-a^{2}}\left[\begin{array}{cc}
1 & -a \\
-a & 1
\end{array}\right]\left\{\begin{array}{l}
\mathbf{n}_{0} \cdot\left(\mathbf{x}_{1}-\mathbf{x}_{0}\right) \\
-\mathbf{n}_{1} \cdot\left(\mathbf{x}_{1}-\mathbf{x}_{0}\right)
\end{array}\right\} & (a \neq \pm 1) \\
\frac{\left[\mathbf{n}_{0}, \pm \mathbf{n}_{0}\right]}{2}\left\{\begin{array}{l}
\mathbf{n}_{0} \cdot\left(\mathbf{x}_{1}-\mathbf{x}_{0}\right) \\
\mp \mathbf{n}_{0} \cdot\left(\mathbf{x}_{1}-\mathbf{x}_{0}\right)
\end{array}\right\}=\mathbf{0} & (a= \pm 1)
\end{array},\right.
$$

where $a=\mathbf{n}_{0} \cdot \mathbf{n}_{1}$, is the cosine of the angle between the normal vectors and $\left[\mathbf{n}_{0}, \mathbf{n}_{1}\right]$ represents a matrix with the first column equal to vector $\mathbf{n}_{0}$ and the second equal to vector $\mathbf{n}_{1}$. The above formulation, described to an edge, can be extended to general polygonal patches, such as triangular and quadrilateral patches, assuring $C^{1}$ continuity in the nodes and quasi- $C^{1}$ in the edges between patches.

In the case of a triangular patch, schematically presented in Figure 2 (a), its vertices 
have the position vectors $\mathbf{x}_{00}, \mathbf{x}_{10}$ and $\mathbf{x}_{11}$, and normal vectors $\mathbf{n}_{00}, \mathbf{n}_{10}$ and $\mathbf{n}_{11}$, respectively. The resulting interpolated surface is the triangular Nagata patch defined by the following quadratic polynomial:

$$
\mathbf{S}(\eta, \zeta)=\mathbf{c}_{00}+\mathbf{c}_{10} \eta+\mathbf{c}_{01} \zeta+\mathbf{c}_{11} \eta \zeta+\mathbf{c}_{20} \eta^{2}+\mathbf{c}_{02} \zeta^{2}, \quad \text { with } \quad 0 \leq \zeta \leq \eta \leq 1,
$$

where the six coefficient vectors $\mathbf{c}_{i j}$ are calculated using only the position and normal vectors of the mesh vertices, as follows:

$$
\begin{gathered}
\mathbf{c}_{00}=\mathbf{x}_{00}, \\
\mathbf{c}_{10}=\mathbf{x}_{10}-\mathbf{x}_{00}-\mathbf{c}_{1}, \\
\mathbf{c}_{01}=\mathbf{x}_{11}-\mathbf{x}_{10}+\mathbf{c}_{1}-\mathbf{c}_{3}, \\
\mathbf{c}_{11}=\mathbf{c}_{3}-\mathbf{c}_{1}-\mathbf{c}_{2}, \\
\mathbf{c}_{20}=\mathbf{c}_{1}, \\
\mathbf{c}_{02}=\mathbf{c}_{2},
\end{gathered}
$$

where $\mathbf{c}_{1}, \mathbf{c}_{2}$ and $\mathbf{c}_{3}$ are the vectors defined by Equation (8) for the edges $\left(\mathbf{x}_{00}, \mathbf{x}_{10}\right),\left(\mathbf{x}_{10}, \mathbf{x}_{11}\right)$ and $\left(\mathbf{x}_{00}, \mathbf{x}_{11}\right)$, respectively.

The quadrilateral patch, exemplified in Figure 2 (b), is interpolated in a similar way as the triangular patch. Consider that the patch has vertices with position vectors $\mathbf{x}_{00}, \mathbf{x}_{10}, \mathbf{x}_{11}$ and $\mathbf{x}_{01}$ and normal vectors $\mathbf{n}_{00}, \mathbf{n}_{10}, \mathbf{n}_{11}$ and $\mathbf{n}_{01}$, respectively. The vertices do not need to be coplanar. Then, the quadrilateral Nagata patch is given by:

$$
\mathbf{S}(\eta, \zeta)=\mathbf{c}_{00}+\mathbf{c}_{10} \eta+\mathbf{c}_{01} \zeta+\mathbf{c}_{11} \eta \zeta+\mathbf{c}_{20} \eta^{2}+\mathbf{c}_{02} \zeta^{2}+\mathbf{c}_{21} \eta^{2} \zeta+\mathbf{c}_{12} \eta \zeta^{2}, \text { with } 0 \leq \eta, \zeta \leq 1,
$$

where the eight coefficient vectors $\mathbf{c}_{i j}$ are calculated using only the position and normal vectors of the mesh vertices, as follows:

$$
\begin{gathered}
\mathbf{c}_{00}=\mathbf{x}_{00}, \\
\mathbf{c}_{10}=\mathbf{x}_{10}-\mathbf{x}_{00}-\mathbf{c}_{1}, \\
\mathbf{c}_{01}=\mathbf{x}_{01}-\mathbf{x}_{00}-\mathbf{c}_{4}, \\
\mathbf{c}_{11}=\mathbf{x}_{11}-\mathbf{x}_{10}-\mathbf{x}_{01}+\mathbf{x}_{00}+\mathbf{c}_{1}-\mathbf{c}_{2}-\mathbf{c}_{3}+\mathbf{c}_{4}, \\
\mathbf{c}_{20}=\mathbf{c}_{1}, \\
\mathbf{c}_{02}=\mathbf{c}_{4}, \\
\mathbf{c}_{21}=\mathbf{c}_{3}-\mathbf{c}_{1}, \\
\mathbf{c}_{12}=\mathbf{c}_{2}-\mathbf{c}_{4},
\end{gathered}
$$

where $\mathbf{c}_{1}, \mathbf{c}_{2}, \mathbf{c}_{3}$ and $\mathbf{c}_{4}$ are the vectors defined by Equation (8) for the edges $\left(\mathbf{x}_{00}, \mathbf{x}_{10}\right)$, $\left(\mathbf{x}_{10}, \mathbf{x}_{11}\right),\left(\mathbf{x}_{01}, \mathbf{x}_{11}\right)$ and $\left(\mathbf{x}_{00}, \mathbf{x}_{01}\right)$, respectively. The above formulation can be extended for other polygonal patch types [6]. Nevertheless, triangular and quadrilateral elements are the most commonly used in mesh generators to describe surfaces. 


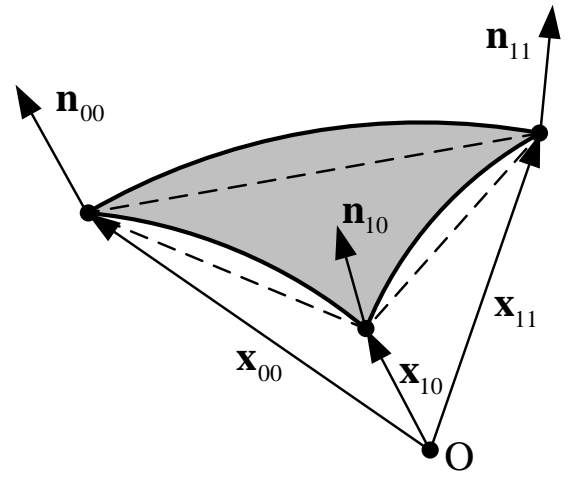

(a)

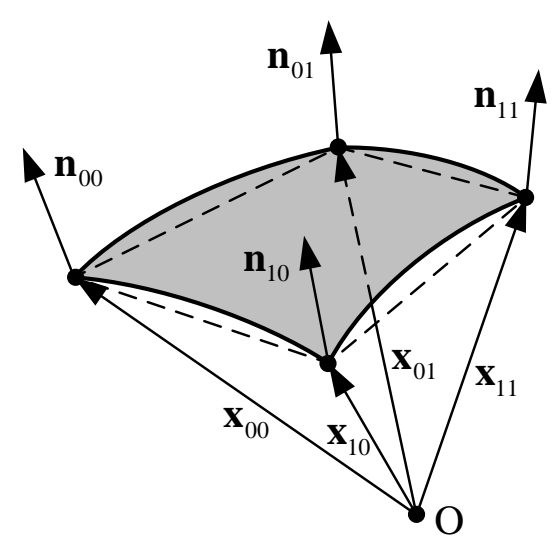

(b)

Figure 2. Nagata patch interpolation: (a) triangular patch; (b) quadrilateral patch.

\subsection{Equivalence between biquadratic Bézier and quadrilateral Nagata patches}

Since the quadrilateral Nagata patch is a quadratic degree surface in both parametric directions and presents the same surface domain as a Bézier patch, it is possible to establish a mathematical equivalency between the biquadratic Bézier patch and the quadrilateral Nagata patch. This equivalence is performed considering the Bézier form, which uses control points in its definition. Thus, according to Equation (1), a biquadratic Bézier patch is given by:

$$
\mathbf{S}(\eta, \zeta)=\sum_{i=0}^{2} \sum_{j=0}^{2} B_{i, 2}(\eta) B_{j, 2}(\zeta) \mathbf{P}_{i j}
$$

where the Bernstein polynomials of degree two are defined using both Equation (2) and (3). The expansion of the sum in the above equation leads to the following polynomial:

$$
\begin{aligned}
\mathbf{S}(\eta, \zeta) & =\mathbf{P}_{00}+\left(2 \mathbf{P}_{10}-2 \mathbf{P}_{00}\right) \eta+\left(2 \mathbf{P}_{01}-2 \mathbf{P}_{00}\right) \zeta+\left(4 \mathbf{P}_{00}-4 \mathbf{P}_{01}-4 \mathbf{P}_{10}+4 \mathbf{P}_{11}\right) \eta \zeta+ \\
& +\left(\mathbf{P}_{00}-2 \mathbf{P}_{10}+\mathbf{P}_{20}\right) \eta^{2}+\left(\mathbf{P}_{00}-2 \mathbf{P}_{01}+\mathbf{P}_{02}\right) \zeta^{2}+ \\
& +\left(2 \mathbf{P}_{01}-2 \mathbf{P}_{00}+4 \mathbf{P}_{10}-4 \mathbf{P}_{11}-2 \mathbf{P}_{20}+2 \mathbf{P}_{21}\right) \eta^{2} \zeta+ \\
& +\left(4 \mathbf{P}_{01}-2 \mathbf{P}_{00}-2 \mathbf{P}_{02}+2 \mathbf{P}_{10}-4 \mathbf{P}_{11}+2 \mathbf{P}_{12}\right) \eta \zeta^{2}+ \\
& +\left(\mathbf{P}_{00}-2 \mathbf{P}_{01}+\mathbf{P}_{02}-2 \mathbf{P}_{10}+4 \mathbf{P}_{11}-2 \mathbf{P}_{12}+\mathbf{P}_{20}+2 \mathbf{P}_{21}+\mathbf{P}_{22}\right) \eta^{2} \zeta^{2}
\end{aligned}
$$

In order to compare this equation with the one corresponding to the quadrilateral Nagata patch, presented in Equation (11), the arrangement of the sums was performed in the same way. Hence, matching each corresponding term of the two equations leads to a system of equations, which associates the Bézier control points to the Nagata patch coefficients. Solving this system of equations by means of isolating the Bézier control points, leads to following solution: 


$$
\begin{gathered}
\mathbf{P}_{00}=\mathbf{c}_{00}, \\
\mathbf{P}_{10}=\mathbf{c}_{00}+\frac{1}{2} \mathbf{c}_{10}, \\
\mathbf{P}_{20}=\mathbf{c}_{00}+\mathbf{c}_{10}+\mathbf{c}_{20}, \\
\mathbf{P}_{01}=\mathbf{c}_{00}+\frac{1}{2} \mathbf{c}_{01}, \\
\mathbf{P}_{11}=\mathbf{c}_{00}+\frac{1}{2}\left(\mathbf{c}_{10}+\mathbf{c}_{01}\right)+\frac{1}{4} \mathbf{c}_{11}, \\
\mathbf{P}_{21}=\mathbf{c}_{00}+\mathbf{c}_{10}+\mathbf{c}_{20} \frac{1}{2}\left(\mathbf{c}_{01}+\mathbf{c}_{11}+\mathbf{c}_{21}\right), \\
\mathbf{P}_{02}=\mathbf{c}_{00}+\mathbf{c}_{01}+\mathbf{c}_{02}, \\
\mathbf{P}_{12}=\mathbf{c}_{00}+\mathbf{c}_{01}+\mathbf{c}_{02}+\frac{1}{2}\left(\mathbf{c}_{10}+\mathbf{c}_{11}+\mathbf{c}_{12}\right), \\
\mathbf{P}_{22}=\mathbf{c}_{00}+\mathbf{c}_{10}+\mathbf{c}_{01}+\mathbf{c}_{11}+\mathbf{c}_{20}+\mathbf{c}_{02}+\mathbf{c}_{21}+\mathbf{c}_{12} .
\end{gathered}
$$

This formulation allows converting a quadrilateral Nagata patch into the corresponding biquadratic Bézier patch, with exactly the same geometry. It should be noted that the vice-versa approach can also be useful, i.e. obtain the Nagata coefficients from the control points. However, this operation can lead to a discrepancy between both surfaces since the last term of Equation (14) is omitted in the Nagata patch formulation (see Equation (11)).

\section{CONTACT SEARCH ALGORITHM}

The number of patches required to define each tool involved in the process to be simulated depends on the geometric complexity of the model. The aim of the contact search algorithm is to identify, for each node of the deformable body candidate to establish contact, both the tool patch and the exactly position where contact can occur. The contact search algorithm must be simultaneously accurate and efficient in order to quickly identify all potential contact zones [10]. Thus, usually it is divided in two phases: global and local search. The global search algorithm must identify all potential contact patches, while the local contact search algorithm must identify the position where the node will establish contact. The main motivation for global contact search is to minimize the number of operations of the local search algorithm, which is usually computationally more expensive [9].

The contact search algorithm implemented in DD3IMP solver, for Bézier patches, has been continuously tested and optimized [10]. This algorithm exploits the special features of parametric Bézier patches to ensure proper and efficient contact detection. Thus, the implementation and tuning of the contact search algorithm for Nagata patches was performed using as reference the knowledge acquired with Bézier patches. The main difference between both types of parametric patches lies in the number of patches needed to properly describe the tools geometry. Since the Nagata patch is only a quadratic surface, typically it is necessary to use more patches to attain the same accuracy as using Bézier. Whatever the parametric description adopted, the first phase of the global search algorithm consists in associating a predefined set of tools to each node, based only on the orientation of each patch outward normal vector to 
the blank. The remaining phases of the global contact search algorithm, for both types of parametric patch, are described in detail in the next subsections.

\subsection{Global contact search for Bézier}

The remaining phases of the global contact search algorithm implemented in DD3IMP for Bézier patches are the following: (a) construction of a uniform grid of points on each patch; (b) evaluation of the distances between each node of the deformable body and the points of the grid, and (c) selection of ten candidate patches to perform the local contact search. The dimension of the grid constructed over each patch is determined based on the maximum degree, $P D_{\eta}^{\max }$ and $P D_{\zeta}^{\max }$, for each parametric patch direction $\eta$ and $\zeta$, respectively. Therefore, in the beginning of the numerical simulation those values are determined for the tool in analysis. Thus, the number of grid divisions, $G D$, in each direction is determined taking into account the maximum patch degree, according to the following expressions:

$$
G D_{\eta}=2 P D_{\eta}^{\max } \text { and } G D_{\zeta}=2 P D_{\zeta}^{\max }
$$

Note that the total number of divisions for each Bézier patch is given by $\left\{\left(G D_{\eta}\right)\left(G D_{\zeta}\right)\right\}$, leading a total number of grid points equal to $\left\{\left(G D_{\eta}+1\right)\left(G D_{\zeta}+1\right)\right\}$, for each patch. This grid is uniformly distributed in the both local coordinates of the patch $(\eta, \zeta)$. Note that this grid of points is calculated only at the beginning of the simulation and updated when the tool position changes. As previously mentioned, in the first phase of the global search a set of tools is selected for each node of the deformable body candidate to establish contact. Thus, the third phase consists in evaluating the distance between the node and every point of each grid, created on the patches which compose the set of candidates. This process is repeated, in each incremental tool displacement, in order to determine at the beginning of each increment the ten Bézier patches closest to the each node, which was not in contact in the previous increment. Hence, for each node it is stored both the identification number of the ten selected patches, as well as the local coordinates of the closest grid point, which will be used as initial approximation for the projection algorithm (local contact search).

\subsection{Global contact search for Nagata}

The global contact search algorithm implemented in DD3IMP solver for Nagata patches was developed with the same philosophy previously applied to Bézier patches. However, the special features of the Nagata patch were explored, such as mesh connectivity of the patches and coordinates of the tool nodes, in order to improve the efficiency and robustness of the implemented algorithm. Thus, the selection of the candidate patches to contact with each node of the deformable body is based mainly in geometric considerations between this node and the tools discretization. The remaining phases of the developed algorithm can also be divided into: (a) selection of a certain number of tool nodes closer to the deformable body node and application of inverse mesh connectivity in order to select their corresponding patches; (b) 
construction of a uniform grid of points on each patch of every tool, and (c) selection of the candidate patches for local contact search based on the distance between the deformable body node and every grid point.

For each node of the deformable body candidate to establish contact, the second phase of the global search algorithm consists in evaluating the distance between the deformable body node and every node of the set of tools, predefined at the beginning of global search. Thus, for each tool contained within this set, the selected number of closest tool nodes is given by:

$$
N=5+\operatorname{int}\left(0.015 R t_{\max }{ }^{2}\right),
$$

where int () is the function that converts a real number into a integer and $R t_{\max }$ is the maximum ratio between the maximum and minimum edge length of each finite element used in tool definition. This strategy allows selecting a different number of closest tool nodes for each tool, guaranteeing that this number depends on the tool mesh topology. After that, the patches that share these tool nodes are selected through the mesh connectivity. However, the number of patches selected using this strategy can be excessive to directly apply the local contact search algorithm. Hence, in order to reduce the number of patches for the local search, the third phase of the global search comprises the creation of a grid of points on each patch, which compose the tool surfaces, such as in the case of Bézier patches. The grid of points is composed by the same number of divisions in each parametric direction since the Nagata patch degree is the same in both directions. Thus, the number of grid divisions in each parametric direction is given by:

$$
G D=\operatorname{int}\left(\max \left(2, \frac{R_{\max }}{10}\right)\right)
$$

where $\max ()$ is the maximum function and $R_{\max }$ is the maximum ratio between the maximum and minimum edge length of each finite element, used in every tool surface discretization. Note that this third phase in performed only at the beginning of each numerical simulation, since the coordinates of grid points are updated based on the tool displacement in each increment. The last phase is identical to the one implemented for Bézier surfaces, where the ten Nagata patches closest to each deformable body node are selected through the distance between the node and every point of each grid. This process is performed in each incremental tool displacement. For each node, it is stored both the identification number of the ten selected patches, as well as the local coordinates of the closest grid point, which will be used as initial approximation for the projection algorithm (local contact search).

\subsection{Local contact search: projection algorithm}

Most of the finite element codes dealing with large deformation contact problems are based on the so called master/slave contact strategy combined with the node-to-segment contact search strategy [16]. This means that the nodes of the deformable body (slave nodes) are prohibited from penetrating the surface of the rigid body (master surface). Then, for each 
node of the deformable body $\mathbf{x}^{\text {def }}$, the objective of the local contact search is to select one patch, from all candidates previously determined in the global search, in order to determine the reference position $\mathbf{x}^{\text {ref }}$ where contact can be established. Presently, the projection algorithm is used in DD3IMP code to find the reference position for each node of the deformable body candidate to establish contact. This algorithm evaluates the reference position $\mathbf{x}^{\text {ref }}$, based on the orthogonal projection of the deformable body node $\mathbf{x}^{\text {def }}$ on the tool surface placed in the actual position, minimizing the distance between the node and the reference position, as shown in Figure 3 [9].

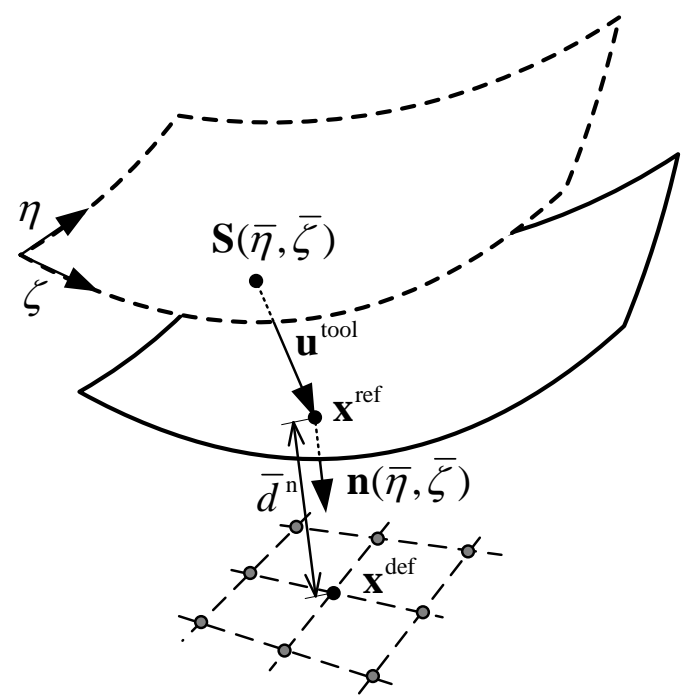

Figure 3. Orthogonal projection of a generic node of the deformable body on a patch composing the tool surface.

The projection algorithm is applied in each iteration of every tool displacement increment. For each node of the deformable body $\mathbf{x}^{\text {def }}$, the implicit coordinates of the reference position $\mathbf{x}^{\text {ref }}$ and the normal distance $\bar{d}^{\mathrm{n}}$ are evaluated through the solution of the following system of equations:

$$
\mathbf{F}^{\mathrm{Proj}}\left(\bar{\eta}, \bar{\zeta}, \bar{d}^{\mathrm{n}}\right)=\mathbf{S}(\bar{\eta}, \bar{\zeta})+\mathbf{u}^{\mathrm{tool}}+\bar{d}^{\mathrm{n}} \mathbf{n}(\bar{\eta}, \bar{\zeta})-\mathbf{x}^{\mathrm{def}}=\mathbf{0},
$$

where $\mathbf{S}(\eta, \zeta)$ represents any point on the selected patch (Bézier or Nagata), $\mathbf{u}^{\text {tool }}$ is the tool displacement from the beginning until the actual position and $\mathbf{n}(\eta, \zeta)$ is the outward patch normal. Since the patch definition $\mathbf{S}(\eta, \zeta)$ and its outward normal vector $\mathbf{n}(\eta, \zeta)$ are nonlinear functions of the parametric coordinates $(\eta, \zeta)$, a iterative method is mandatory to solve the problem. The Newton-Raphson algorithm is used to solve this system of equations, which can be summarized as follows for the $i+1$ iteration:

$$
\overline{\mathbf{a}}^{(i+1)}=\overline{\mathbf{a}}^{(i)}-\left.\left[\left.\nabla \mathbf{F}^{\text {Proj }}\right|^{(i)}\right]^{-1} \mathbf{F}^{\text {Proj }}\right|^{(i)}, \quad \text { with } \quad \overline{\mathbf{a}} \equiv\left[\bar{\eta}, \bar{\zeta}, \bar{d}^{\mathrm{n}}\right]^{\mathrm{T}},
$$

where $\overline{\mathbf{a}}$ is the solution vector and $\nabla \mathbf{F}^{\text {Proj }}$ is the Jacobian matrix of the system of equations presented in (19). In this study, the convergence criterion is based on the simultaneous satisfaction of the two following conditions: 


$$
\left\{\begin{array}{c}
\left|\overline{\mathbf{a}}^{(i+1)}-\overline{\mathbf{a}}^{(i)}\right| \leq \varepsilon_{\mathrm{a}}^{\text {Conv }} \\
\left|\mathbf{F}^{\text {Proj }}\right|^{(i)} \mid \leq \varepsilon_{\mathrm{F}}^{\text {Conv }}
\end{array},\right.
$$

where $\varepsilon_{\mathrm{a}}^{\text {Conv }}$ and $\varepsilon_{\mathrm{F}}^{\text {Conv }}$ are predefined threshold values. Note that the system of equations (19) is solved for every patch preselected in the global contact search. In case of multiple solutions the algorithm selects the solution with the minimum normal distance $\bar{d}^{\mathrm{n}}$ value.

The Jacobian matrix $\nabla \mathbf{F}^{\text {Proj }}$ presented in system of equation (20) is defined as:

$$
\nabla \mathbf{F}^{\text {Proj }}\left(\eta, \zeta, d^{\mathrm{n}}\right)=\left[\frac{\partial}{\partial \eta}, \frac{\partial}{\partial \zeta}, \frac{\partial}{\partial d^{\mathrm{n}}}\right] \mathbf{F}^{\text {Proj }}\left(\eta, \zeta, d^{\mathrm{n}}\right) .
$$

Thus, in order to determine the Jacobian matrix it is necessary to calculate the partial derivatives of $\mathbf{F}^{\text {Proj }}$, which result in the partial derivatives of both the patch and its normal vector, with respect to the local coordinates. The unit normal vector can be defined as a function of the patch local coordinates through the cross product of its first partial derivatives, as follows:

$$
\mathbf{n}(\eta, \zeta)=\frac{\mathbf{S}_{\eta}(\eta, \zeta) \times \mathbf{S}_{\zeta}(\eta, \zeta)}{\left|\mathbf{S}_{\eta}(\eta, \zeta) \times \mathbf{S}_{\zeta}(\eta, \zeta)\right|}
$$

where $\mathbf{S}_{\eta}(\eta, \zeta)$ and $\mathbf{S}_{\zeta}(\eta, \zeta)$ are the first partial derivatives of the patch with respect to $\eta$ and $\zeta$, respectively. Both first partial derivatives of the patches can be easily derived from the Equation (4) for Bézier patches and Equations (9) and (11) for triangular and quadrilateral Nagata patches, respectively. On the other hand, the gradient of the normal vector with respect to local coordinates of the patch is evaluated using the Weingarten formula [3].

\section{COMPUTATIONAL PERFORMANCE}

This section presents the influence of the global contact search algorithms implemented on the performance of the local contact search algorithm. In order to compare the developed algorithms, the numerical simulation of a deep drawing process was selected. Different tool models are employed in the numerical simulations, which are composed of Nagata patches interpolations of both structured and unstructured discretizations. The punch force evolution and the sheet thickness are selected to compare the numerical results obtained with all tool models tested. Finally, the algorithms performance is evaluated in terms of computational cost.

\subsection{Cross tool deep drawing process}

The numerical simulation of the cross tool deep drawing process was the selected example to analyze the performance of the developed contact search algorithms, implemented in the DD3IMP solver. This problem involves three rigid tools: punch, blank holder and die, leading to a process decomposed into three phases: blank-holder clamping, punch displace- 
ment until $60 \mathrm{~mm}$ and springback. The blank sheet is a square with $250 \mathrm{~mm}$ length and 0.8 $\mathrm{mm}$ thickness. Due to geometrical and material symmetry conditions, only one quarter of the global model is simulated, as shown in Figure 4 (a). The mechanical properties of the mild steel considered for the blank sheet are presented in Table 1. A friction coefficient of 0.03 is defined for the contact between the tools and the sheet, while the blank holder force used as a constant value of $72.5 \mathrm{kN}$. This process parameter was selected in order to prevent the occurrence of necking, tearing and wrinkling defects in the final part. The blank sheet was discretized with a regular mesh composed by 7688 hexahedron solid finite elements, with two element layers in the thickness direction. All simulations were carried out in a computer equipped with Intel® ${ }^{\circledR}$ Core ${ }^{\mathrm{TM}}$ i7-950 $(3.07 \mathrm{GHz}) \mathrm{CPU}$ and the Windows7 Professional (64bits platform) operating system.

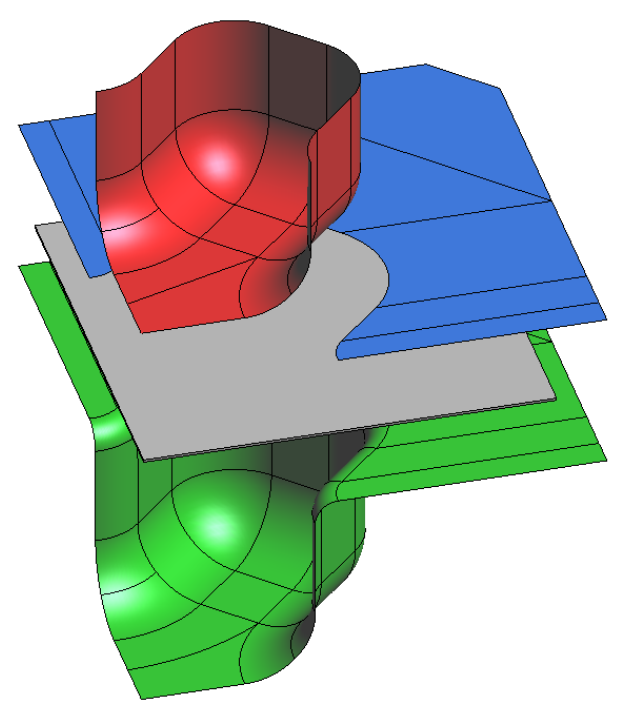

(a)

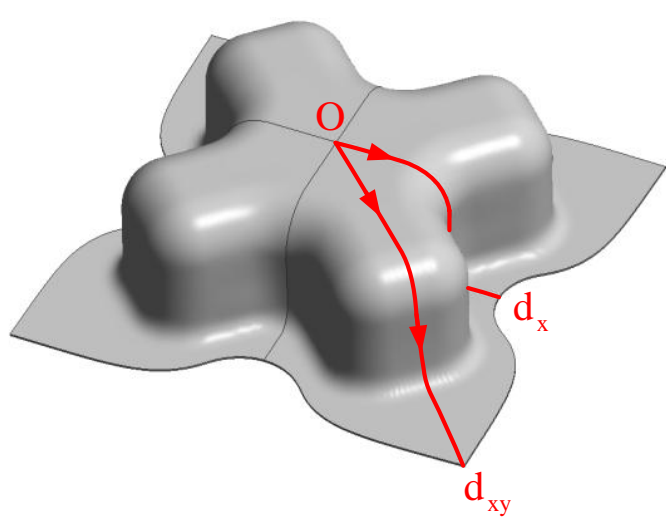

(b)

Figure 4. Cross tool deep drawing process: (a) surface tools described by Bézier patches; (b) directions selected for thickness measurements in the formed part.

Table 1. Material parameters of mild steel.

\begin{tabular}{ccc}
\hline \multirow{2}{*}{ Elastic Proprieties } & $\begin{array}{c}\text { Swift Hardening Law } \\
\sigma=K\left(\varepsilon_{0}+\bar{\varepsilon}^{\mathrm{p}}\right)^{n}\end{array}$ & Hill'48 Yield Criterion \\
\hline$E=210 \mathrm{GPa}$ & $K=529.5 \mathrm{MPa}$ & $F=0.251$ \\
$v=0.3$ & $\varepsilon_{0}=0.00439$ & $G=0.297$ \\
& $n=0.268$ & $H=0.703$ \\
& $L=M=1.50$ \\
& $N=1.290$ \\
\hline
\end{tabular}

\subsection{Results analysis and discussion}

This section presents the comparison of the numerical results obtained with every tool model tested in this study. The total computational time required to complete each simulation is also studied and compared between tool models. The results obtained from the tool model composed by Bézier patches of arbitrary order (labeled Bézier AO) are assumed as the refer- 
ence results, since it is the model previously validated. The forming tool modeled by Bézier patches is shown in Figure 4 (a), which is composed by a total of 61 patches with a maximum degree of six. Note that the CAD model (IGES file format) required to generate the models composed by Nagata patches was created from the CAD model composed by Bézier patches of arbitrary order, through the STEP file format. In order to evaluate the influence of the number of Nagata patches used in the tool description seven tool models were created. Table 2 summarizes, for each of the seven models studied, the number of patches used as well as the typology and topology of the Nagata patches discretization. The structured discretizations are labeled by "St", while the unstructured are denoted by "Unst". The discretizations composed only of quadrilateral Nagata patches are labeled by "QN" while the label "TN" is used in case of discretizations composed only of triangular Nagata patches. The study considers also one model composed by both types (quadrilateral and triangular) of patches, which are denoted by "MN".

Table 2. Number of Nagata patches used in each forming tool model.

\begin{tabular}{ccccc}
\hline Tool model & Punch & Blank holder & Die & Total \\
\hline St-QN-85 & 28 & 10 & 47 & 85 \\
St-QN-210 & 74 & 16 & 120 & 210 \\
St-QN-589 & 230 & 28 & 331 & 589 \\
St-QN-1241 & 472 & 40 & 729 & 1241 \\
St-MN-226 & 82 & 16 & 128 & 226 \\
Unst-TN-1848 & 471 & 328 & 1049 & 1848 \\
Unst-QN-1103 & 309 & 174 & 620 & 1103 \\
\hline
\end{tabular}

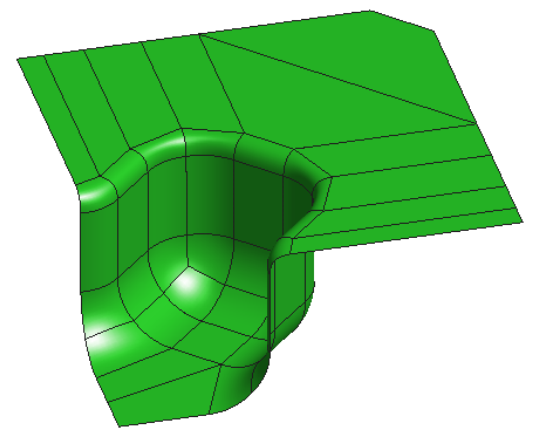

(a)

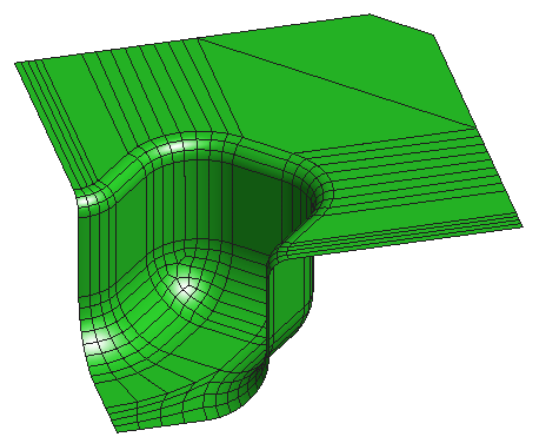

(c)

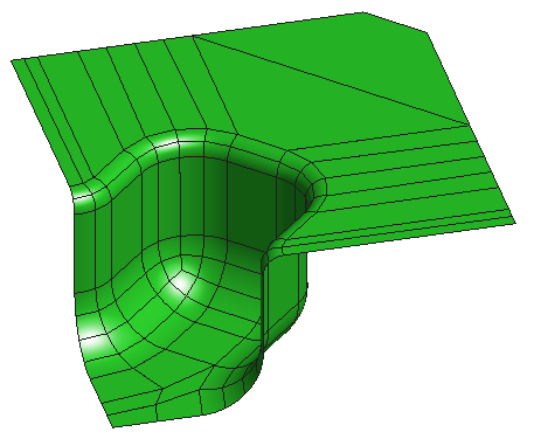

(b)

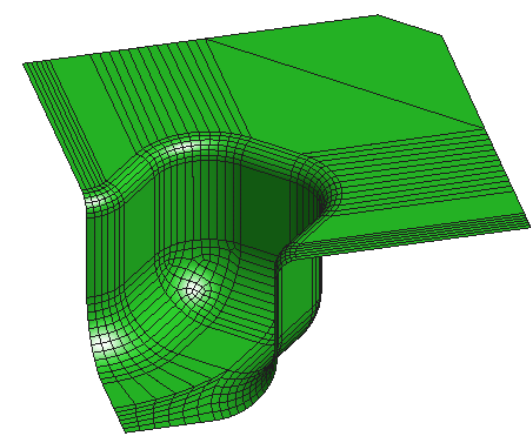

(d)

Figure 5. Die described by a structured discretization of quadrilateral Nagata patches: (a) StQN-85; (b) St-QN-210; (c) St-QN-589; (d) St-QN-1241. 
Figure 5 presents the die discretization for the four models which are described using structured quadrilateral Nagata patches. The punch and blank holder are omitted since they present a similar geometry to the die, as shown in Figure 4 (a), and consequently present a similar patch discretization. The flat areas of the tool are discretized with the minimum number of patches, in order to reduce the total number of required patches to accurately describe the tools geometry. On the other hand, the curved tool areas are discretized with an increasing number of patches, which leads to increasing tool geometry accuracy (minimize the shape error) $[6,7]$. Figure 6 presents the comparison of the force punch evolution for the QN" tool models considered. A slight difference is observed in the punch force evolution for the model "St-QN-85", which is the one presenting the smaller number of patches in its definition. The remaining models have a force evolution similar to the one obtained with tools described by Bézier patches. As shown in Figure 7, the same behavior is observed for the final sheet thickness distribution, measured along both direction presented in Figure 4 (b). The predicted sheet thickness for the tool model composed by a smaller number of patches is smaller than in other tool models, mostly along the direction $d_{x}$, as shown in Figure 7 (a).

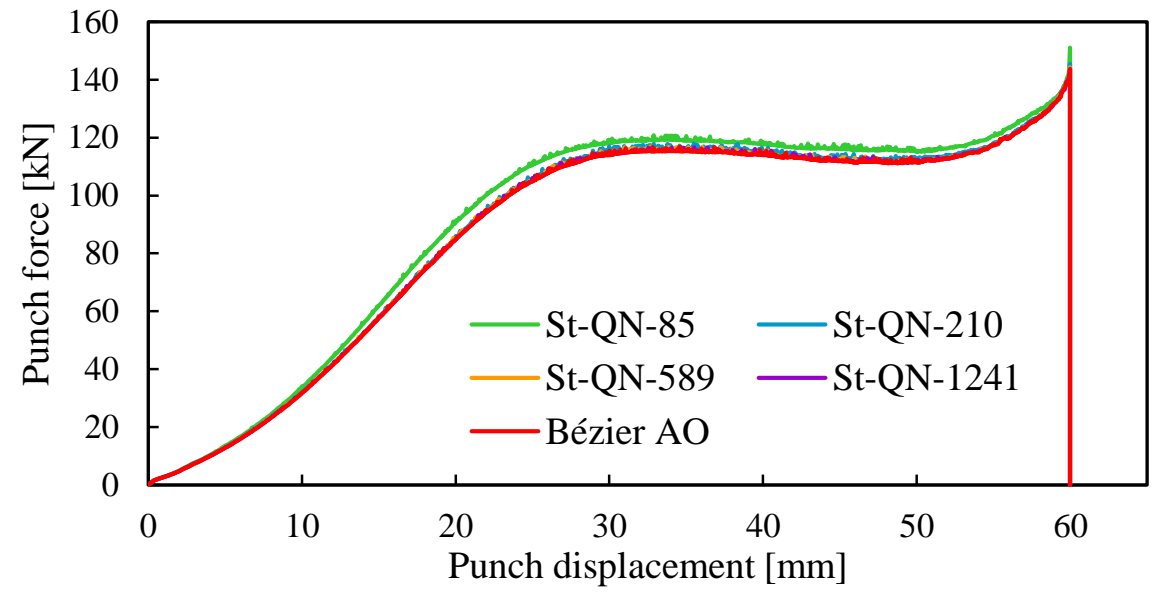

Figure 6. Punch force evolution with its displacement for forming tools composed by structured discretizations of quadrilateral Nagata patches.

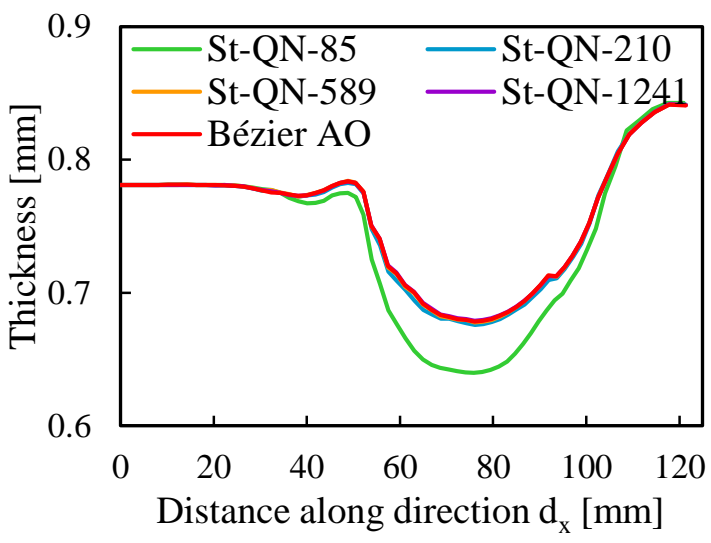

(a)

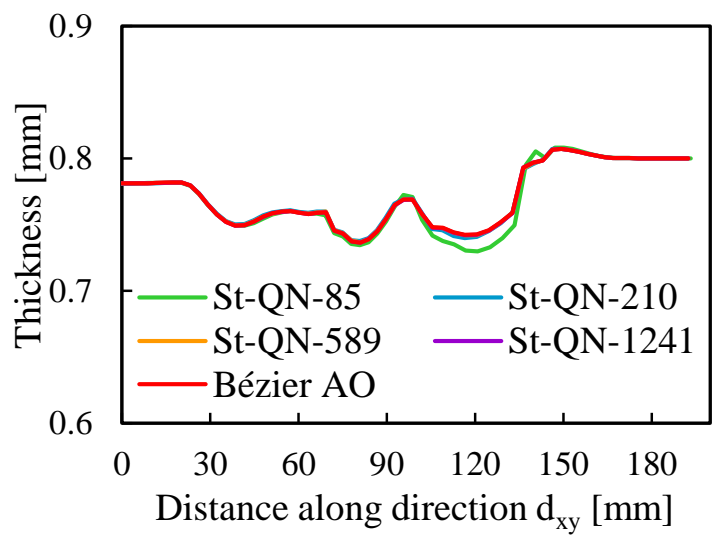

(b)

Figure 7. Thickness distribution at the end of the deep drawing process using forming tools composed by structured discretizations of quadrilateral Nagata patches: (a) along direction $\mathrm{d}_{\mathrm{x}}$; (b) along direction $\mathrm{d}_{\mathrm{xy}}$. 
The discrepancy of the numerical simulation results for the tool model "St-QN-85" is due to the Nagata patch interpolation accuracy. Both the global and local contact search algorithms developed to deal with Nagata patches are efficient in the contact detection for every tool model studied. Therefore, in order to quantify the geometry tool model accuracy, the shape error distribution in each Nagata patch is determined, as follows:

$$
\delta_{\text {Shape }}=\left(\mathbf{P}_{\text {Nagata }}-\mathbf{P}_{\mathrm{CAD}}\right) \cdot \mathbf{n}_{\mathrm{CAD}},
$$

where $\mathbf{P}_{\text {Nagata }}$ is the position vector of a generic point on the patch, $\mathbf{P}_{\mathrm{CAD}}$ is the corresponding orthogonal projection on the CAD model (IGES file composed by trimmed NURBS surfaces) and $\mathbf{n}_{\mathrm{CAD}}$ is the unit surface normal vector at the projected point. Figure 8 presents the comparison of the shape error distribution between both two die models with less number of patches (St-QN-85 and St-QN-210). In both models, the maximum shape error attained is located in the hyperbolic section of the torus that corresponds to a fillet with radius of $7 \mathrm{~mm}$. Nevertheless, the maximum value attained for the "St-QN-210" model is one magnitude order lower than the one obtained with the "St-QN-85". Hence, models with a geometric error as the one obtained with the"St-QN-85" model lead to different numerical results and should be avoided.

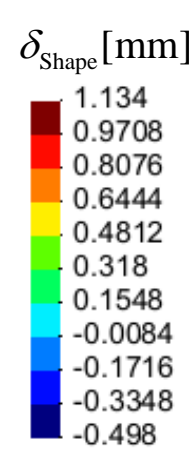

Figure 8. Shape error dist

(a)

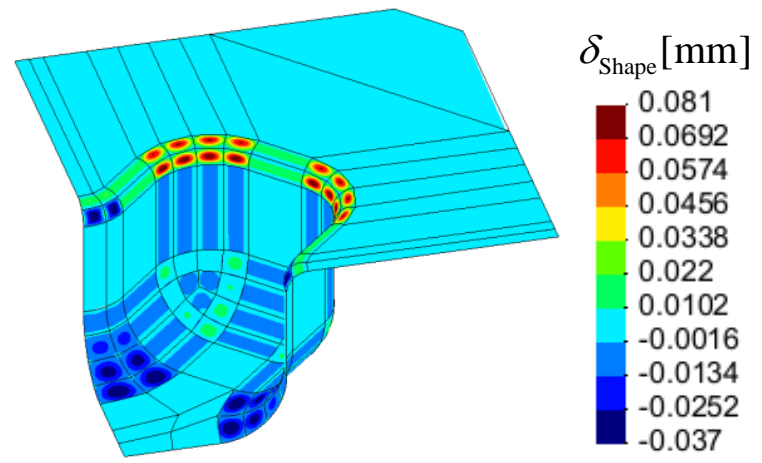

(b)

(a) St-QN-85; (b) St-

QN-210.

As described in section 2.3, it is possible to establish the equivalence between the quadrilateral Nagata patches and the biquadratic Bézier patches. Therefore, in order to test the efficiency and robustness of the algorithm already implemented for Bézier patches when applied to a larger number of patches, the four "QN" Nagata models were converted into models composed by biquadratic Bézier patches. Since the geometry of both types of models is exactly the same, the numerical results obtained should be the same in case of correct contact detection. The use of the global contact search algorithm presented in section 2.1, to deal with the contact treatment evolving Bézier patches, leads to the same punch force and thickness evolution as the one obtained with Nagata patches, presented in Figure 6 and Figure 7, respectively.

The tool models previously studied are composed only by quadrilateral Nagata patches. However, the discretization of 3-sides surfaces is easier with triangular elements, which 
will originate triangular Nagata patches after interpolation. It should be mentioned that unstructured discretizations are simpler to generate. Nevertheless, the distorted generated elements (quadrilateral or triangular) can lead to problems in the Nagata patches tool geometry accuracy.

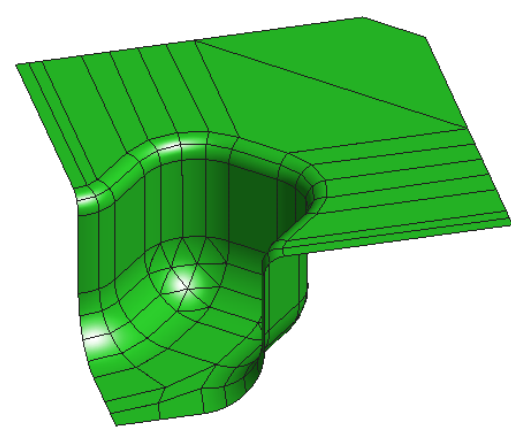

(a)

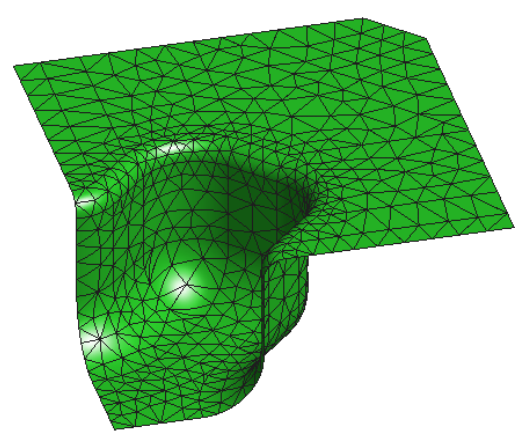

(b)

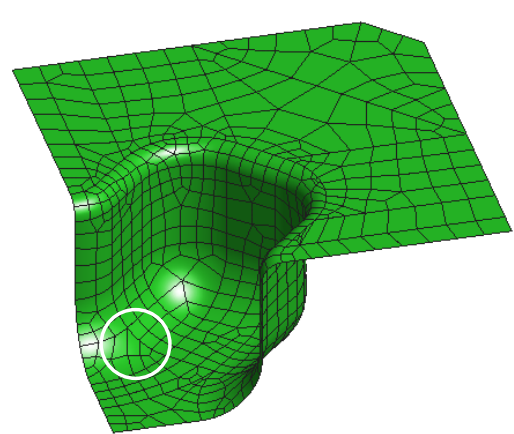

(c)

Figure 9. Discretization of the die with Nagata patches for the model: (a) St-MN-226; (b) Unst-TN-1848; (c) Unst-QN-1103.

The tool model comprising a structured discretization with both triangular and quadrilateral Nagata patches is shown in Figure 9 (a). The only difference to the "St-QN-210" model presented in Figure 5 (b) is the discretization of the 3-side surfaces with triangular patches. In order to complete the analysis of the effect of tool discretization, two unstructured discretizations were built, one composed only by triangular patches (Figure 9 (b)) and other one with quadrilateral patches (Figure 9 (c)). Figure 10 presents the comparison of the punch force evolution for the three tool models shown in Figure 9. The punch force evolution for the model "Unst-QN-1103" presents a high increase in the final part of the forming process, attaining the maximum value of $340 \mathrm{kN}$. Note that the punch force scale was clipped in order to highlight the region of the reference results. The punch force peak for the "Unst-QN-1103" is due to the type of tools used in the process, i.e. the punch geometry is obtained offsetting the die geometry with a value equal to the initial sheet thickness. Hence, at the end of the deep drawing process, the gap between the punch and die should be constant and equal to initial sheet thickness. However, the tool model composed by an unstructured discretization of quadrilateral Nagata patches presents excessive shape error in the region of the die indicated in Figure 9 (b) with a circle. Thus, the gap between the die and the punch at end of the process is considerable smaller than the initial sheet thickness and, consequently, the punch force increase abruptly. 


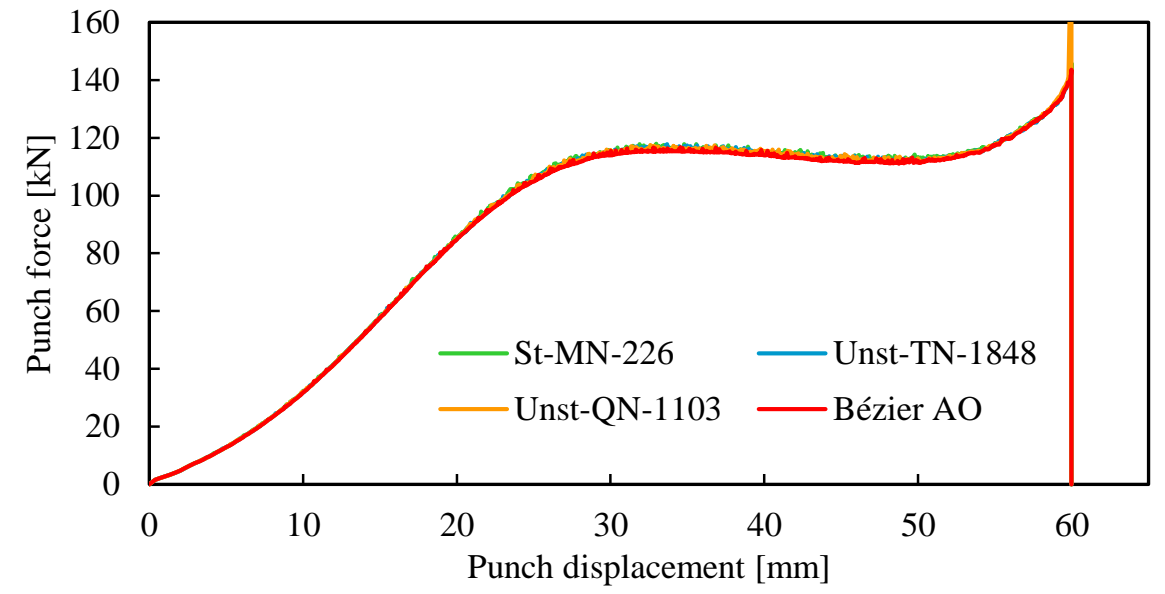

Figure 10. Comparison of the punch force evolution for forming tools composed by unstructured discretizations of Nagata patches.

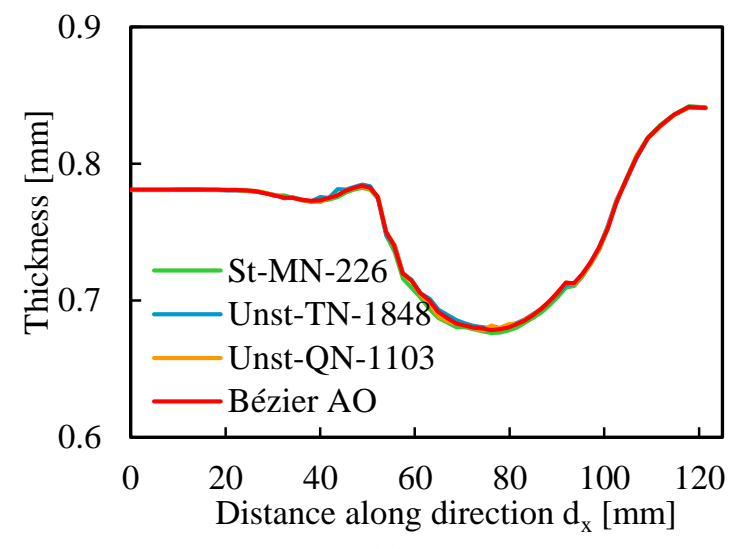

(a)

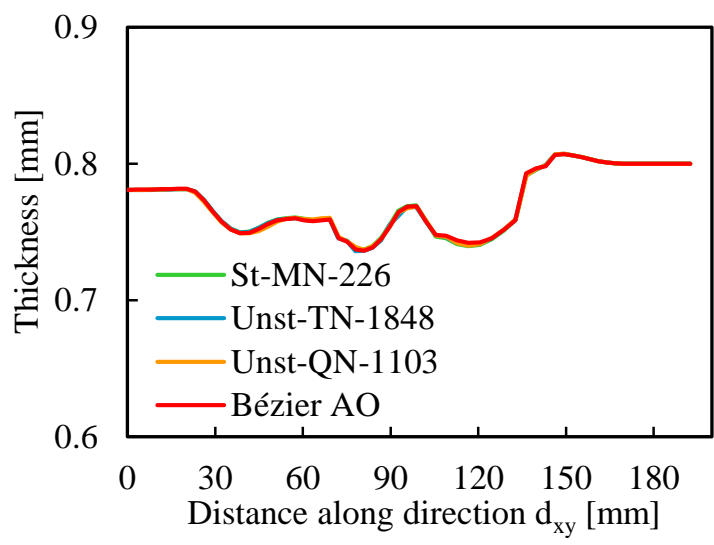

(b)

Figure 11. Thickness distribution at the end of process using forming tools composed by unstructured discretizations: (a) along direction $\mathrm{d}_{\mathrm{x}}$; (b) along direction $\mathrm{d}_{\mathrm{xy}}$.

Figure 11 shows the thickness evolution along both directions indicated in Figure 4 (b), for the tool models composed by unstructured Nagata patches. In contrast with the punch force evolution, the thickness distribution at the end of the process is correctly predicted for all tool models. In fact, the two directions where the thickness evolution is studied are located far from the die region with excessive shape error, as visible by the comparison between Figure 4 (b) and Figure 9 (c). These results confirm the local effect of the tool shape error distribution.

In addition to the numerical results accuracy, the computation time becomes an increasingly important factor in the efficiency of any simulation code. Figure 12 presents the total computational time obtained for each of the tool models used in the numerical simulation. Globally, the computational time is almost the same for all tool models studied. However, the global contact search algorithm implemented for Nagata patches seems more insensitive to the total number of patches used in the tool surfaces description, when compared with the one applied for Bézier patches. The tool models composed by Nagata patches have a computational cost slightly inferior to the one obtained with the reference result (Bézier AO), except the "Unst-TN-1848" model. 


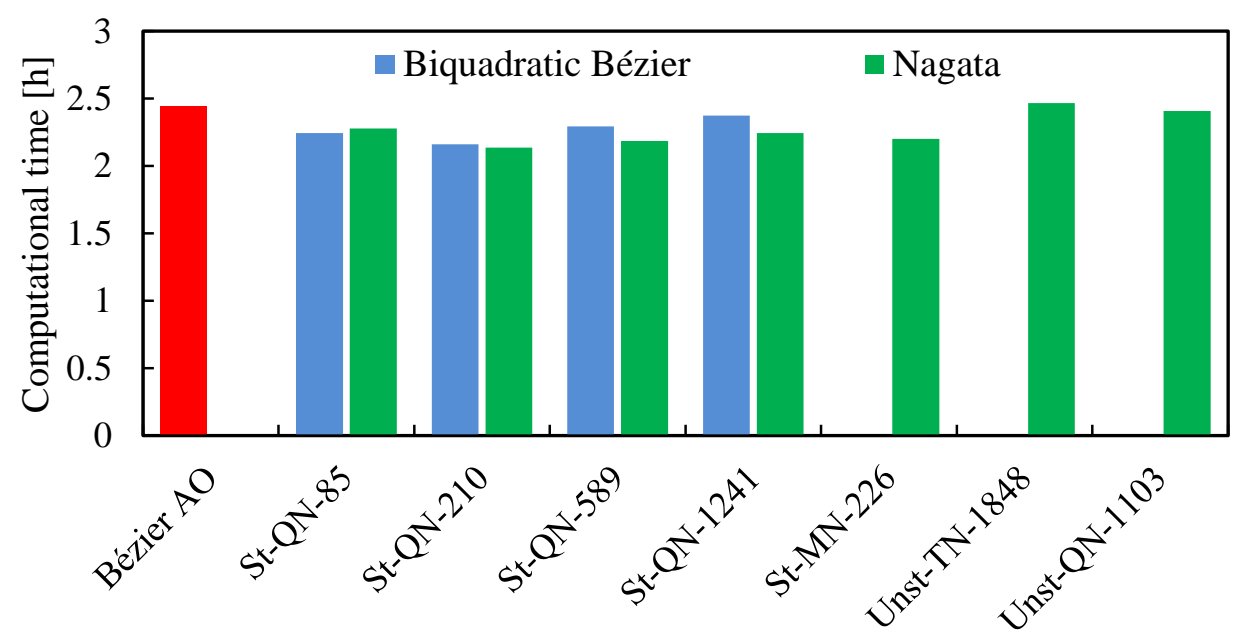

Figure 12. Comparison of the computational time for all tool models studied.

\section{CONCLUSIONS}

The new global contact search algorithm implemented in DD3IMP solver to deal with Nagata patches in tool surface description was successfully validated with the numerical simulation of a forming process involving complex tool geometry. The numerical simulation of the cross tool deep drawing process was the selected one for this study in order to highlight the robustness and efficiency of the new developed algorithm in complex forming processes. Several tool surface discretizations were performed with both triangular and quadrilateral Nagata patches, as well structured and unstructured discretizations. The punch force evolution and thickness distribution, at the end of the process, were evaluated and compared for all tool models studied. The results obtained with Nagata patches show that for a wide range of tool surface discretizations, all numerical results are in agreement with those previously obtained with the global search algorithm implemented for Bézier patches of arbitrary order. In terms of computational cost, both algorithms present the same behavior. The use of Nagata patches in the tool description is more versatile than Bézier, since they can be easily generated from any combination of CAD and mesh generation software.

\section{Acknowledgements}

The authors gratefully acknowledge the financial support of the Portuguese Foundation for Science and Technology (FCT) under Project PTDC/EME-TME/103350/2008 by FEDER through the program QREN (COMPETE: FCOMP-01-0124-FEDER-010301). The first author is also grateful to the FCT for the PhD grant SFRH/BD/69140/2010.

\section{REFERENCES}

[1] Farin G., Curves and Surfaces for Computer-Aided Geometric Design: A Practical Guide. Academic Press, 1997. 
[2] Hama T., Nagata T., Teodosiu C., Makinouchi A., Takuda H., "Finite-element simulation of springback in sheet metal forming using local interpolation for tool surfaces". Int. J. Mech. Sci. 50, 175-192, 2008.

[3] Konyukhov A., Schweizerhof K., " Covariant description for frictional contact problems". Comput. Mech. 35, 190-213, 2005.

[4] Lengiewicz J., Stupkiewicz S., Rodic T., Korelc J., Sustaric P., Contact smoothing in sensitivity analysis and optimization of multi-body contact problems. Oñate E., Owen D. R. J. Eds. VIII International Conference on Computational Plasticity, Barcelona, 2005.

[5] Menezes L. F., Teodosiu C., "Three-dimensional numerical simulation of the deepdrawing process using solid finite elements". J. Mater. Process. Tech. 97, 100-106, 2000.

[6] Nagata T., "Simple local interpolation of surfaces using normal vectors". Comput. Aided Geom. Des. 22, 327-347, 2005.

[7] Neto D. M., Oliveira M. C., Alves J. L., Menezes L. F., Local Interpolation for Tools Surface Description. Barlat F., Moon Y. H., Lee M. G. Eds. NUMIFORM 2010, Pohang, 2010.

[8] Neto D. M., Oliveira M. C., Menezes L. F., Alves J. L., Evaluating the vertex normal vector in polyhedral mesh using the IGES file format. Andrade-Campos A., Lopes N., Valente R. A. F., Varum H. Eds. First ECCOMAS Young Investigators Conference, Aveiro, 2012.

[9] Oliveira M. C., Alves J. L., Menezes L. F., "Improvement of a frictional contact algorithm for strongly curved contact problems". Int. J. Numer. Meth. Engng. 58, 2083-2101, 2003.

[10] Oliveira M. C., Alves, J. L., Menezes, L. F., "Algorithms and Strategies for Treatment of Large Deformation Frictional Contact in the Numerical Simulation of Deep Drawing Process". Arch. Comput. Method Eng. 15, 113-162, 2008.

[11] Pietrzak G., Curnier A., "Large deformation frictional contact mechanics: continuum formulation and augmented Lagrangian treatment". Comput. Meth. Appl. Mech. Eng. 177, 351-381, 1999.

[12] Puso M. A., Laursen T. A., "A 3D contact smoothing method using Gregory patches". Int. J. Numer. Meth. Engng. 54, 1161-1194, 2002.

[13] Rogers D. F., An Introduction to NURBS with Historical Perspective, Morgan Kaufmann Publishers, 2001.

[14] Santos A., Makinouchi A., "Contact strategies to deal with different tool descriptions in static explicit FEM of 3-D sheet-metal forming simulation". J. Mater. Process. Tech. 50, 277-291, 1995.

[15] Stadler M., Holzapfel G. A., Korelc J., " $\mathrm{C}^{\mathrm{n}}$ continuous modelling of smooth contact surfaces using NURBS and application to 2D problems". Int. J. Numer. Meth. Engng. 57, 2177-2203, 2003.

[16] Wriggers P., "Finite Element Algorithms for Contact Problems". Arch. Comput. Method Eng. 2, 1-49, 1995.

[17] Wriggers P., Krstulovic-Opara L., Korelc J., "Smooth $\mathrm{C}^{1}$-interpolations for two dimensional frictional problems". Int. J. Numer. Meth. Engng. 51, 1469-1495, 2001.

[18] Zhuang S., Lee M. G., Keum Y. T., Kim J. H., Wagoner R. H., "Improved contact procedure for implicit finite element sheet forming simulation". Int. J. Numer. Meth. Engng. 83, 1759-1779, 2010. 